\title{
Population Dynamics and Non-Hermitian Localization
}

\author{
Karin A. Dahment, David R. Nelson \\ Lyman Laboratory of Physics, Harvard University, Cambrigde, MA, 02138 \\ Nadav M. Shnerb \\ Racah Institute of Physics, Hebrew University, Jerusalem 91904, Israel
}

\begin{abstract}
We review localization with non-Hermitian time evolution as applied to simple models of population biology with spatially varying growth profiles and convection. Convection leads to a constant imaginary vector potential in the Schrödinger-like operator which appears in linearized growth models. We illustrate the basic ideas by reviewing how convection affects the evolution of a population influenced by a simple square well growth profile. Results from discrete lattice growth models in both one and two dimensions are presented. A set of similarity transformations which lead to exact results for the spectrum and winding numbers of eigenfunctions for random growth rates in one dimension is described in detail. We discuss the influence of boundary conditions, and argue that periodic boundary conditions lead to results which are in fact typical of a broad class of growth problems with convection.

PACS numbers: 05.70.Ln,87.22.As,05.40.+j
\end{abstract}

\section{INTRODUCTION}

Bacterial growth in a petri dish, the basic experiment of microbiology, is a familiar but interesting phenomenon. Depending on the nutrient and agar concentration, a variety of intriguing growth patterns have been observed [2 5 . Some regimes can be modeled by diffusion limited aggregation, others by Eden models and still others exhibit ring structures or a two-dimensional modulation in the bacterial density. At high nutrient concentration and low agar density, there is a large regime of simple growth of a circular patch (after point inoculation), described by a Fisher equation [6], and studied experimentally in Ref. [2].

Of course, most bacteria do not live in petri dishes, but rather in inhomogeneous environments characterized by, e.g., spatially varying growth rates and/or diffusion constants. Often, as in the soil after a rain storm (or in a sewage treatment plant), bacterial diffusion and growth are accompanied by convective drift in an aqueous medium through the disorder. By creating artificially modulated growth environments in petri dishes, one can begin to study how bacteria (and other species populations) grow in circumstances more typical of the real world. More generally, the challenges posed by combining inhomogeneous biological processes with various types of fluid flows [7] seem likely to attract considerable interest in the future. The easiest problem to study in the context of bacteria is to determine how fixed spatial inhomogeneities and convective flow affect the simple regime of Fisher equation growth mentioned above.

A delocalization transition in inhomogeneous biological systems has recently been proposed, focusing on a single species continuous growth model, in which the population disperses via diffusion and convection [8]: the Fisher equation [6] for the population number density $c(\mathbf{x}, t)$, generalized to account for convection and an inhomogeneous growth rate, reads [8]

$$
\begin{aligned}
\partial c(\mathbf{x}, t) / \partial t= & D \nabla^{2} c(\mathbf{x}, t)-\mathbf{v} \cdot \nabla c(\mathbf{x}, t) \\
& +U(\mathbf{x}) c(\mathbf{x}, t)-b c^{2}(\mathbf{x}, t),
\end{aligned}
$$

where $D$ is the diffusion constant of the system, $\mathbf{v}$ is the spatially homogeneous convection ("wind") velocity, and $b$ is a phenomenological parameter responsible for the limiting of the concentration $c(\mathbf{x}, t)$ to some maximum saturation value. The growth rate $U(\mathbf{x})$ is a random function which describes a spatially random nutrient concentration, or, for photosynthetic bacteria, an inhomogeneous illumination pattern [8]. If $U(\mathbf{x})$ is constant over the entire sample, then the convection term $-\mathbf{v} \cdot \nabla c(\mathbf{x}, t)$ can be eliminated by a coordinate transformation and has no effect on the growth of the bacteria [9]. Only the introduction of a spatial dependence for the growth rate $U(\mathbf{x})$ makes the convection term interesting.

In this brief review, we summarize recent theoretical results concerning Eq. (1.1). In Section I1, we review results for a "square well" shape for the growth profile $U(x)$ [10]. When linearized about the state of zero population, Eq. (1.1) is related to Schroedinger's equation with a square well potential and analytic results are possible. As we shall see, large enough convective velocities delocalize bound states in the equivalent quantum mechanical problem, and this 
delocalization is accompanied by escape of real growth eigenvalues into the complex plane. This important signature of delocalization is preserved for random growth profiles. A lattice approximation to the continuum Eq. (1.1), useful for treating both square well and random growth profiles, is described in Section III. Exact results and various similarity transformations for the lattice model in one dimension [1] are summarized in Section IV]. The one dimensional lattice model is used to explore the effect of boundary conditions in Section $\square$. In the appendix we give analytical expressions for the spectrum of a two dimensional homogeneous system for two different lattices and the continuum model.

\section{RESULTS FOR SQUARE WELL GROWTH PROFILES}

In the following we review results for equation Eq. (1.1) with a simple "square well" growth profile $U(\mathbf{x})$, imposing a positive growth rate $a$ on an illuminated patch ("oasis"), and a negative growth rate $-\epsilon a$ outside ("desert") [10]:

$$
U(\mathbf{x})= \begin{cases}a, & \text { for }|\mathbf{x}|<\frac{W}{2}, \\ -\epsilon a, & \text { for }|\mathbf{x}| \geq \frac{W}{2},\end{cases}
$$

where $W$ is the diameter of the oasis. Experimentally this situation can be realized using a very simple setup, which illustrates the basic ideas of localization and delocalization and leads to interesting further questions. A one dimensional example is shown in figure 1, where a solution with photosynthetic bacteria in a thin circular pipe, or annular petri dish, is illuminated by a fixed uniform light source through a mask, leading to a "square well" intensity distribution. The mask is moved at a small, constant velocity around the sample to simulate convective flow. (Moving the mask is equivalent to introducing convective flow in the system, up to a change of reference frame [9].) The bacteria are assumed to divide in the brightly illuminated area ("oasis") at a certain rate, but division ceases or proceeds at a greatly reduced rate in the darker region ("desert") outside. As a result, the growth rate in this continuum population dynamics model is positive in the oasis and small (positive or negative) in the surrounding desert region. Using this simple nonlinear growth model, one can study the total number of bacteria expected to survive in the steady state, the shape of their distribution in space and other quantities, as a function of the "convection velocity" of the light source [10].

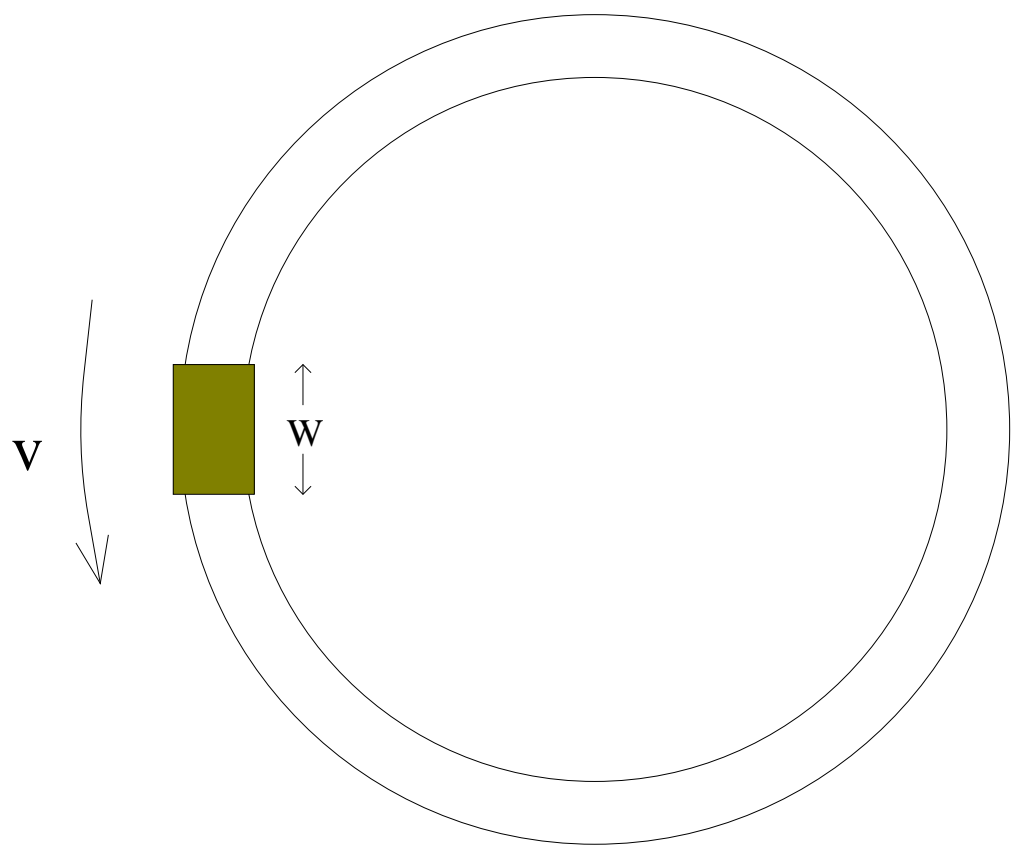

FIG. 1. Experimental setup: a solution with photosynthetic bacteria in a circular pipe or a thin annular track in a petri dish is illuminated only in a small area, while the rest of the sample is either kept dark or illuminated with reduced intensity. The light source (shaded rectangle) is moved slowly around the sample to model convective flow. The bacteria are assumed to divide in the illuminated area ("oasis") at a certain growth rate $a>0$, and die (or grow modestly) in the remaining area ("desert") with growth rate $-\epsilon a$. 
Much can already be learned by linearizing equation (1.1) around $c(\mathbf{x}, t)=0$ :

$$
\partial c(\mathbf{x}, t) / \partial t=\mathcal{L} c(\mathbf{x}, t)
$$

with the linearized growth operator

$$
\mathcal{L}=D \nabla^{2}-\mathbf{v} \cdot \nabla+U(\mathbf{x}) .
$$

The convection velocity $\mathbf{v}$ acts formally like an imaginary vector potential in this Schrödinger-like equation [12]. For $v$ nonzero, $\mathcal{L}$ is non-Hermitian, but it can still be diagonalized by a complete set of right and left eigenvectors $\left\{\phi_{n}^{R}(\mathbf{x})\right\}$ and $\left\{\phi_{n}^{L}(\mathbf{x})\right\}$, with eigenvalues $\Gamma_{n}$, and orthogonality condition

$$
\int d^{d} x \phi_{m}^{L}(\mathbf{x}) \phi_{n}^{R}(\mathbf{x})=\delta_{m, n},
$$

( $d$ is the dimension of the substrate, we focus here on $d=1$ or $d=2$ ). The time evolution of $c(\mathbf{x}, t)$ is then given by

$$
c(\mathbf{x}, t)=\sum_{n} c_{n} \phi_{n}^{R}(\mathbf{x}) \exp \left(\Gamma_{n} t\right),
$$

where the initial conditions and left eigenfunctions determine the coefficients $\left\{c_{n}\right\}$,

$$
c_{n}=\int d^{d} x \phi_{n}^{L}(\mathbf{x}) c(\mathbf{x}, t=0) .
$$

(See Refs. 8] and 10] for a discussion of how the results from the linearized problem can be used to treat the full nonlinear system.)

Fig. 2 shows the complex eigenvalue spectrum associated with Eq. (2.3) for a one-dimensional system with the potential (2.1) and periodic boundary conditions, at four different values of the convection velocity $v$. In Ref. 10] details of the derivation of these results are given. At zero velocity, $\mathcal{L}$ is Hermitian and all eigenvalues $\Gamma_{n}$ are real. There are bound states (discrete spectrum) and extended or delocalized states (continuous spectrum). Because $\mathcal{L}$ resembles the negative of a quantum mechanical Hamiltonian, bound states have the highest eigenvalues (growth rates), in contrast to the usual situation in quantum mechanics. At finite velocities, all delocalized states, except the uppermost, acquire a complex eigenvalue. According to Eq. (2.5), states with positive real part of the eigenvalue $\left(\operatorname{Re} \Gamma_{n}>0\right)$ grow exponentially with time, states with negative real part $\left(\operatorname{Re} \Gamma_{n}<0\right)$ decrease exponentially with time. In a large one dimensional system the "mobility edge" [13], which we define to be the eigenvalue of the fastest growing delocalized state, (i.e. the rightmost eigenvalue in the complex parabolas of figure 2), is located for one dimensional systems of size $L$ at the overall average growth rate

$$
\Gamma^{*}=\langle U\rangle \equiv \int_{0}^{L} d x U(x) / L \simeq-\epsilon a,
$$

In Fig. 2, the eigenvalues of the localized states compose the discrete, real spectrum to the right of the mobility edge. With increasing velocity these localized eigenvalues move uniformly to the left by an amount $v^{2} / 4 D$, and successively enter the continuous delocalized spectrum through the mobility edge, which remains fixed. The parabola also broadens in the vertical direction: the imaginary parts of the eigenvalues of the delocalized states grow by an amount proportional to $v$. A given localized right eigenfunction $\phi_{n}^{R}$ undergoes a "delocalization transition" when the velocity reaches a corresponding critical delocalization velocity $v=v_{n}^{*}$, at which its eigenvalue $\Gamma_{n}$ has been shifted so far to the left that it just touches $\Gamma^{*}$. At higher velocities it joins the parabola of eigenvalues describing a continuum of delocalized states. The ease with which such a delocalization transition can be observed experimentally depends on whether there are growing delocalized eigenstates in the system, i.e. whether the mobility edge has a positive real value or not. 


\section{Spectrum for different drift velocities}

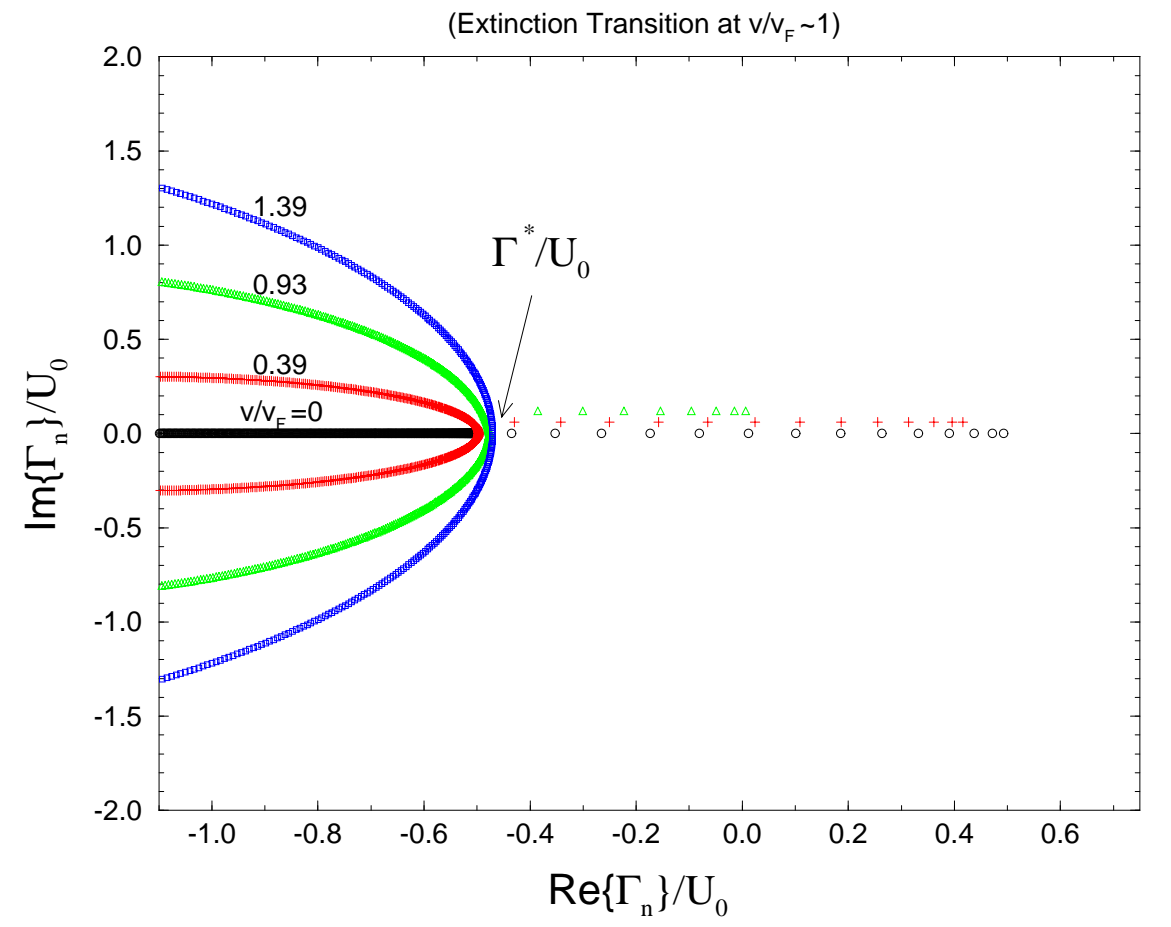

FIG. 2. Complex nonhermitian eigenvalue spectra (normalized by the difference of the growth rates inside and outside the oasis $U_{0} \equiv a+\epsilon a=1$ ) at velocities above and below the extinction transition. The spectra are extracted from numerical simulations of a lattice approximation to the continuum described in Section [II], for a one dimensional system of 1000 sites. In units such that the lattice constant $\ell_{0}=1$, we have oasis width $W=20$ sites, diffusion constant $D=0.3 U_{0} \ell_{0}^{2}$, with growth rate $-\epsilon a=-0.5 U_{0}$ in the desert and $a=+0.5 U_{0}$ in the oasis, so that the average growth rate is $-0.48 U_{0}$ (which is equal to the mobility edge $\Gamma^{*}$ up to finite size effects). The velocity parameters (in units of the Fisher wave velocity in the oasis $v_{F}=2 \sqrt{a D}$ [6]) are $v / v_{F} \sim 0$ (circles), $v / v_{F} \sim 0.39$ (crosses), $v / v_{F} \sim 0.93$ (triangles), and $v / v_{F} \sim 1.39$ (squares). The point spectra are slightly offset in the vertical direction so as to be able to distinguish the eigenvalues of the localized states for different velocities. As the convection velocity increases, the mobility edge remains fixed, and the parabola of the delocalized eigenvalues opens up as $v / v_{F}$ is increased. The real, localized eigenvalues move to the left for higher velocities. When $v / v_{F} \geq 1.39$ all states are delocalized.

We distinguish three cases, $\langle U\rangle\langle 0,\langle U\rangle>0$, and $\langle U\rangle \simeq 0$ :

(1) In a large "deadly" desert $(\langle U\rangle \simeq-\epsilon a<0)$ all delocalized states die out, because the mobility edge lies to the left of the origin, as in Fig. 2. The growth rate of each localized eigenstate $\phi_{n}^{R}$ then becomes negative at a corresponding "extinction" velocity $v_{n c}$ which is smaller than the corresponding delocalization velocity $v_{n}^{*}$. Thus, as convection is increased, the population dies out before it can delocalize. Total extinction occurs when the eigenvalue of the localized "ground state" (fastest growing eigenfunction of $\mathcal{L}$ ) passes through the origin, i.e. when $v=v_{0 c}$ (with $v_{0 c}>v_{n c}$ for all $n>0$. The total bacterial population of the steady state in the corresponding nonlinear problem goes to zero linearly with $v-v_{0 c}[10]$.

(2) If the average growth rate $\langle U\rangle$ is positive (i.e for a small enough desert or a small positive growth rate in an infinite desert), the mobility edge lies to the right of the origin and the delocalization transition can indeed be observed at $v=v_{0}^{*}$ where the "ground state" becomes delocalized. One expects to see universal behavior near this delocalization transition, since there is a diverging correlation length in the system, which renders microscopic details 
irrelevant for certain quantities. For example, the correlation length $\xi$ of the spatial density distribution scales as $\xi \sim 1 /\left(v-v_{0}^{*}\right)^{\nu}$ with a universal exponent $\nu=1[10]$.

(3) A special (universal) behavior is expected for the spatially average growth rate $\langle U\rangle=0$. In this case the delocalization and extinction velocities coincide. Fig. 3 summarizes the different scenarios in a sketch of the phase diagram for large systems with fixed well depth $U_{0} \equiv a+\epsilon a$, obtained by tuning the drift velocity, and the average growth rate $\langle U\rangle \simeq-\epsilon a$. Also shown in Fig. 3 is a horizontal transition line at $\langle U\rangle=0$ separating a small velocity region $(\epsilon<0)$ where localized modes dominate the steady state bacterial population, from one $(\epsilon>0)$ containing a mixture of localized and extended states. It is of course also possible to drive a population extinct at zero velocity simply by lowering the average growth rate. This special transition at $\langle U\rangle=-U_{c}$ is indicated at the bottom of figure 3 .

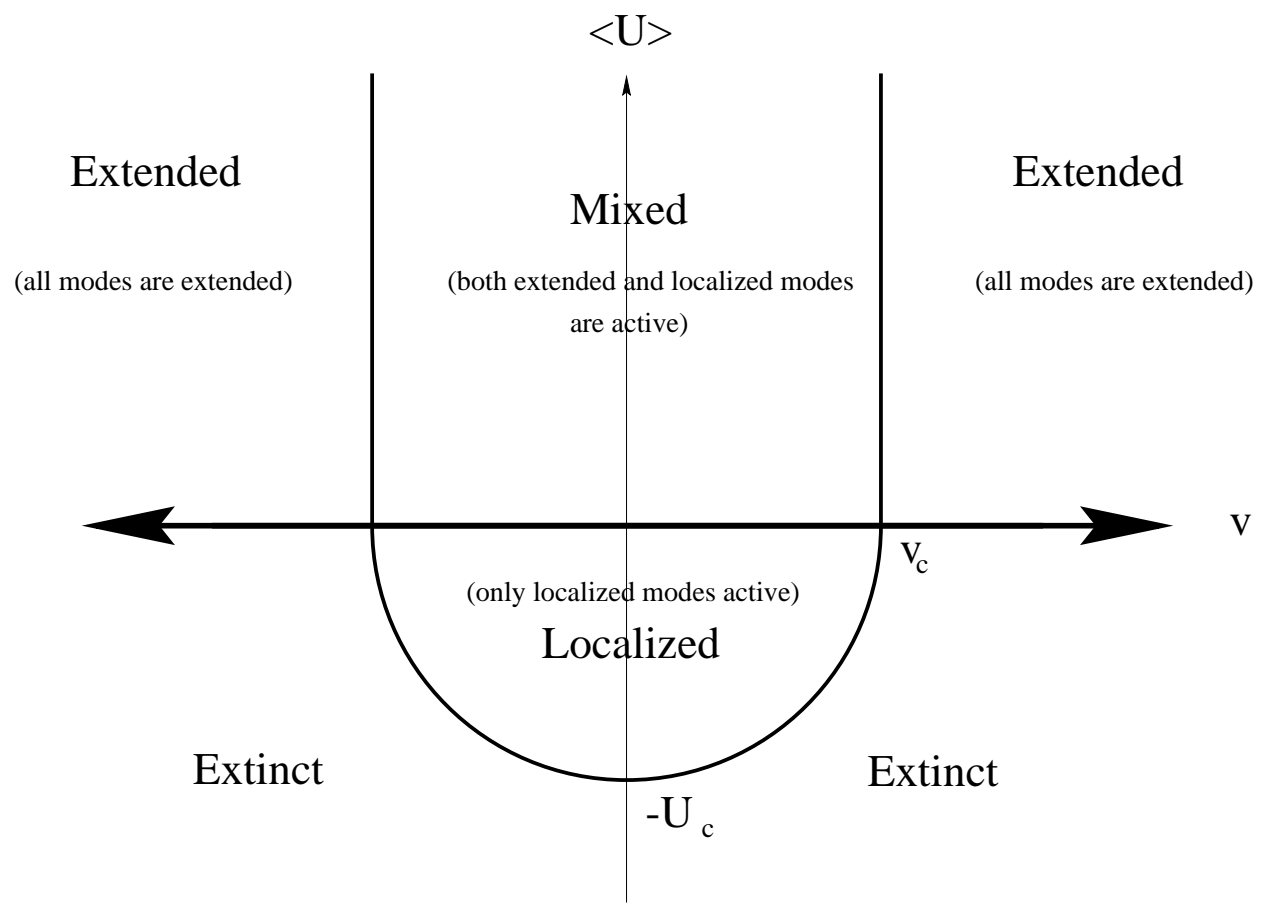

FIG. 3. Schematic phase diagram in one dimension for infinite system size, as a function of average growth rate $\langle U\rangle$ and convection velocity $v$ for fixed "well depth" $U_{0} \equiv a+\epsilon a$. For a deep well $\left(U_{0} \gg D / W^{2}\right)$ the extinction transition out of the localized phase occurs when $v=0$ for $\langle U\rangle=-U_{c}$, where $U_{c} \simeq U_{0}$. The diagram shows that if the growth rate is negative outside and inside the oasis (i.e. $\langle U\rangle<-U_{c}$ ), then the only possible state is extinction at any velocity. If there is positive growth inside the oasis, but negative outside, a localized population can survive in the oasis, but only for small enough wind velocities $v$. Extended states are present for a small positive growth rate in the desert $(\langle U\rangle>0)$. In this case localized and delocalized states coexist for small velocities ("mixed phase"), while at large velocities all eigenstates are extended. The ground state becomes delocalized at the critical velocity $v_{c}$ which marks the phase boundary between the mixed and the extended phase.

\section{LATTICE MODEL OF GROWTH AND CONVECTION}

As discussed in Refs. [8] and [10], analytic expressions for eigenfunctions and eigenvalues obtained from an analysis of a linearized growth problem can be used to study the nonlinear steady state provided only a few unstable growing modes are present. In more general situations, however, a numerical analysis of the full nonlinear Eq. (1.1) may be necessary. Numerical work also provides insights into situations where the growth profile is random, as opposed to the simple square well considered in Section [1]. We review here a lattice approximation to the continuum nonlinear model which is very useful for obtaining numerical results. It was used in Ref. 10] to obtain growth spectra like those shown in figure 2 to confirm and extend analytic results obtained in one dimension. Here, we apply the lattice model to some illustrative two dimensional growth problems with convection.

A discrete lattice approximation (discrete in space but continuous in time) to Eq. (1.1) in $d$ dimensions reads [8] 


$$
\begin{aligned}
\frac{d c_{\mathbf{x}}(t)}{d t}= & w \sum_{\nu=1}^{d}\left[e^{\mathbf{g} \cdot \mathbf{e}_{\nu}} c_{\mathbf{x}+\mathbf{e}_{\nu}}(t)+e^{-\mathbf{g} \cdot \mathbf{e}_{\nu}} c_{\mathbf{x}-\mathbf{e}_{\nu}}(t)-2 \cosh \left(\mathbf{g} \cdot \mathbf{e}_{\nu}\right) c_{\mathbf{x}}(t)\right] \\
& +U(\mathbf{x}) c_{\mathbf{x}}(t)-b c_{\mathbf{x}}^{2}(t)
\end{aligned}
$$

where $c_{\mathbf{x}}(t)$ is the species population at the sites $\{\mathbf{x}\}$ of a hypercubic lattice with lattice constant $\ell_{0}$, and the $\left\{\mathbf{e}_{\nu}\right\}$ are unit lattice vectors. Furthermore, $w \simeq D / \ell_{0}^{2}$, where $D$ is the diffusion constant of the corresponding continuum model, and $g \simeq-v \ell_{0} /(2 D)$, where $v$ is the convective flow rate of the continuum model. $U(\mathbf{x})$ and $b$ have the same interpretation as in the continuum model (1.1). The subtraction in the first term insures that $c_{\mathbf{x}}(t)$ is conserved $\left(\frac{d}{d t} \sum_{\mathbf{x}} c_{\mathbf{x}}(t)=0\right)$ if $U(x)=b=0$. When linearized about $c_{\mathbf{x}} \equiv 0$, Eq. (3.1) may be written

$$
\frac{d c_{\mathbf{x}}(t)}{d t}=\sum_{\mathbf{x}^{\prime}} \tilde{\mathcal{L}}\left(\mathbf{x}, \mathbf{x}^{\prime}\right) c_{\mathbf{x}^{\prime}}(t)
$$

where the discrete Liouville operator $\tilde{\mathcal{L}}$ is the matrix

$$
\begin{aligned}
\tilde{\mathcal{L}}= & w \sum_{\mathbf{x}} \sum_{\nu=1}^{d}\left[e^{-\mathbf{g} \cdot \mathbf{e}_{\nu}}\left|\mathbf{x}+\mathbf{e}_{\nu}\right\rangle\left\langle\mathbf{x}\left|+e^{\mathbf{g} \cdot \mathbf{e}_{\nu}}\right| \mathbf{x}\right\rangle\left\langle\mathbf{x}+\mathbf{e}_{\nu}\right|\right] \\
& +\sum_{\mathbf{x}}[A+U(\mathbf{x})]|\mathbf{x}\rangle\langle\mathbf{x}|
\end{aligned}
$$

with $A=-2 w \sum_{\nu=1}^{d} \cosh \left(\mathbf{g} \cdot \mathbf{e}_{\nu}\right)$. Typical spectra with a square well growth profile for a one dimensional 1000-site model were shown in Fig. 2 for several values of $g \propto v[10]$. Parameter values and energies studied were chosen to give a good approximation to the continuum.

For two dimensional systems, a triangular lattice (see Eq. (3.5)) is actually a better approximation to the continuum than a square lattice, because it minimizes artifacts caused by preferred lattice directions. Using the unit lattice vectors $\mathbf{e}_{1}, \mathbf{e}_{2}, \mathbf{e}_{3}$ of Fig. [1 to define the Laplacian, one finds [14] via a Taylor's series expansion

$$
\begin{aligned}
\frac{2}{3 \ell_{0}^{2}} \sum_{\nu=1}^{3}\left[c_{\mathbf{x}+\mathbf{e}_{\nu}}(t)+c_{\mathbf{x}-\mathbf{e}_{\nu}}(t)-2 c_{\mathbf{x}}(t)\right] & \\
& =\nabla^{2} c_{\mathbf{x}}(t)+\frac{1}{16} \ell_{0}^{2}\left(\nabla^{4} c_{\mathbf{x}}(t)\right)+O\left(\ell_{0}^{4}\right) .
\end{aligned}
$$

Using the lattice vectors $\mathbf{e}_{1}$, and $\mathbf{e}_{2}$ of a square lattice gives a similar result, but the fourth order terms in that case are $\ell_{0}^{4}\left(\partial^{4} / \partial x^{4}+\partial^{4} / \partial y^{4}\right) c_{\mathbf{x}}(t) / 12$ which cannot be expressed in terms of the Laplacian of $c_{\mathbf{x}}(t)[14$. 


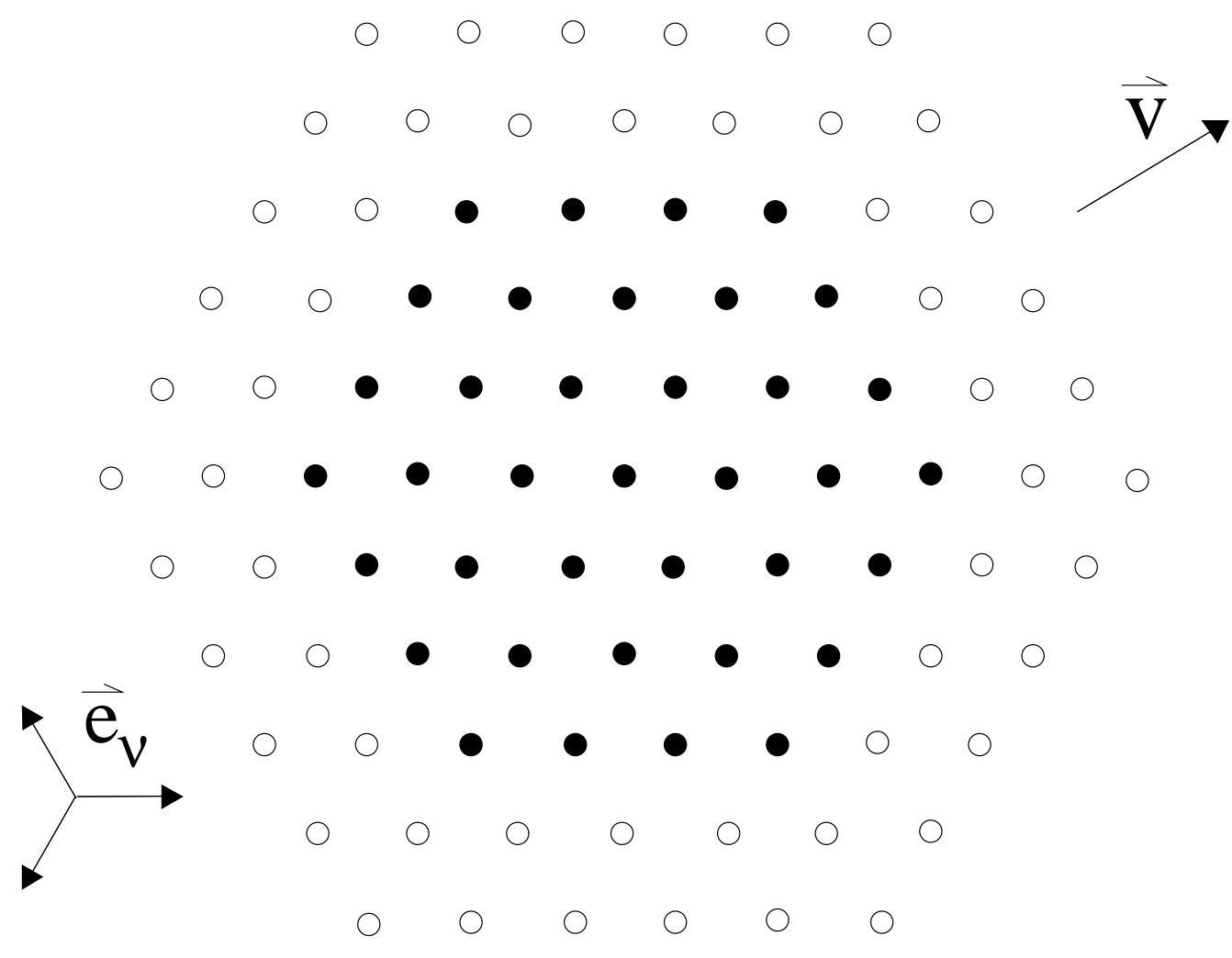

FIG. 4. Hexagonal oasis (filled circles) surrounded by a desert (empty circles), in 2 dimensions on a triangular lattice, with periodic boundary conditions. The inset in the lower left corner of the figure displays the three basis vectors $\mathbf{e}_{\nu}$ of the lattice. The arrow in the upper right corner of the figure indicates the $30^{\circ}$ direction of the convection velocity $\mathbf{v}$ relative to the horizontal axis. (This direction renders a better approximation to the continuum than for example a direction along one of the three lattice vectors $\mathbf{e}_{\nu}$.)

Fig. 5 shows the eigenvalue spectra at finite convection velocity $\mathbf{v}$ for an oasis in a desert for two different lattices, for the square lattice, with $\mathbf{v}$ along the $x$ axis(a), and along the $45^{\circ}$-axis (b), and for the triangular lattice (see Eq. (3.5)) with $\mathbf{v}$ along the $x$ axis (c) and along the $30^{\circ}$-axis (d) as in Fig 4 . States localized near the oasis again appear as a string of real eigenvalues above a pattern of complex eigenvalues representing delocalized eigenfunctions. Although the ellipsoidal pattern of extended state eigenvalues is an artifact of the lattice, the upper edge can be a good approximation to the continuum if the direction is chosen appropriately. Directions coinciding with "lattice planes" lead to spurious features in the delocalized part of the spectrum. Spectral artifacts are particularly noticeable for the square lattice, when $\mathbf{v}$ is along one of the lattice directions (a). Note also, that for the square lattice the ellipse of complex eigenvalues is mirror symmetric with respect to the two coordinate axes through the center of the ellipse. For neither the triangular lattice, nor the continuum model, there is such a mirror symmetry with respect to the imaginary axis in the complex spectrum. An easy check is given by an analytic computation of the spectrum for a homogeneous growth rate in the system. The results are cited in the appendix. 


\section{$\operatorname{Im}\{\Gamma\} / \mathrm{U}_{0}$ versus $\operatorname{Re}\{\Gamma\} / \mathrm{U}_{0}$ for square and triangular lattices}
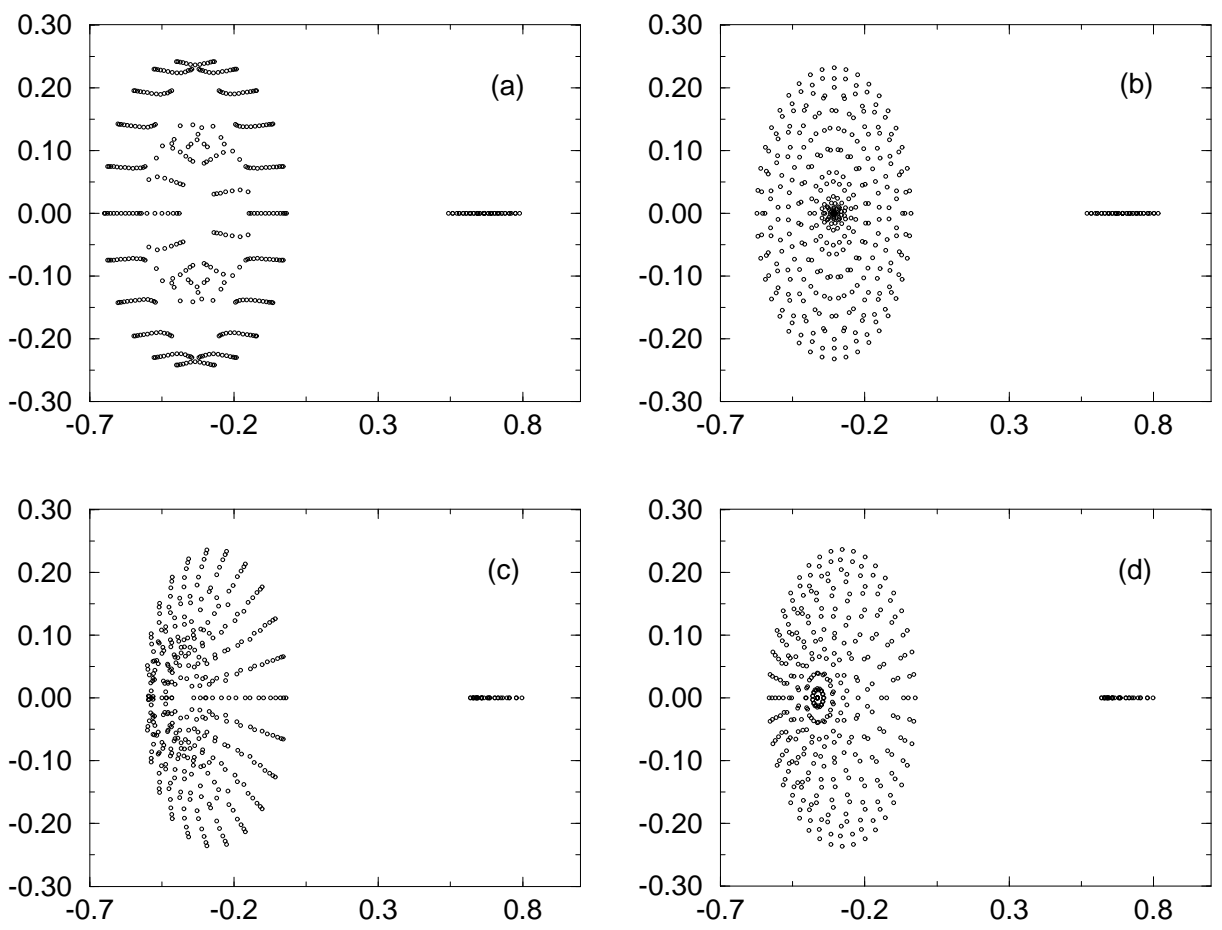

FIG. 5. Complex nonhermitian eigenvalue spectra for a two dimensional oasis in a desert, using different lattices. The model parameters (with $\ell_{0} \equiv 1$ ) are $D=w=1, v / v_{F}=0.37, a=29.5 w$, and $\epsilon a=0.5 w$. (a) and (b) show results for a square lattice with 400 sites and a square oasis with 36 sites, with periodic boundary conditions. In (a) the velocity $\mathbf{v}$ is directed along the $x$-axis, which is one of the two preferred lattice directions. In (b) the velocity is directed along the diagonal (45 -axis). (c) and (d) show the results for a triangular lattice with 400 sites, a hexagonal oasis with 37 sites, with periodic boundary conditions, as shown in Fig 4 . In (c) the velocity is directed along the $x$ axis, which coincides with the direction of one of the lattice vectors $\mathbf{e}_{\nu}$. In (d) the velocity is directed along the $30^{\circ}$-axis, as indicated in Fig 4 . (The eigenvalues are normalized by the difference of the growth rates inside and outside the oasis $U_{0}=a+\epsilon a=30 \mathrm{w}$.)

We now use a triangular lattice to study the delocalization transition in two dimensions. For a triangular lattice the sum in Eq. (3.3) over $\nu$ is replaced by a sum over three unit lattice vectors illustrated in Fig 1 . One obtains

$$
\begin{aligned}
\tilde{\mathcal{L}}= & \frac{2 w}{3} \sum_{\mathbf{x}} \sum_{\nu=1}^{3}\left[e^{-\mathbf{g} \cdot \mathbf{e}_{\nu}}\left|\mathbf{x}+\mathbf{e}_{\nu}\right\rangle\left\langle\mathbf{x}\left|+e^{\mathbf{g} \cdot \mathbf{e}_{\nu}}\right| \mathbf{x}\right\rangle\left\langle\mathbf{x}+\mathbf{e}_{\nu}\right|\right] \\
& +\sum_{\mathbf{x}}[A+U(\mathbf{x})]|\mathbf{x}\rangle\langle\mathbf{x}|
\end{aligned}
$$

with $A=-\frac{4 w}{3} \sum_{\nu=1}^{3} \cosh \left(\mathbf{g} \cdot \mathbf{e}_{\nu}\right)$. Fig. 6 shows lattice simulation results for six spectra at different convection velocities for the linearized two dimensional system with a hexagonal oasis, and periodic boundary conditions. The convection velocity is oriented along the $30^{\circ}$ axis relative to the lattice, as shown in figure 6 . 


\section{Spectra for an oasis in 2 dimensions}

( $\operatorname{Im}\{\Gamma\} / \mathrm{U}_{0}$ vs $\operatorname{Re}\{\Gamma\} / \mathrm{U}_{0}$ for 6 different convection velocities )
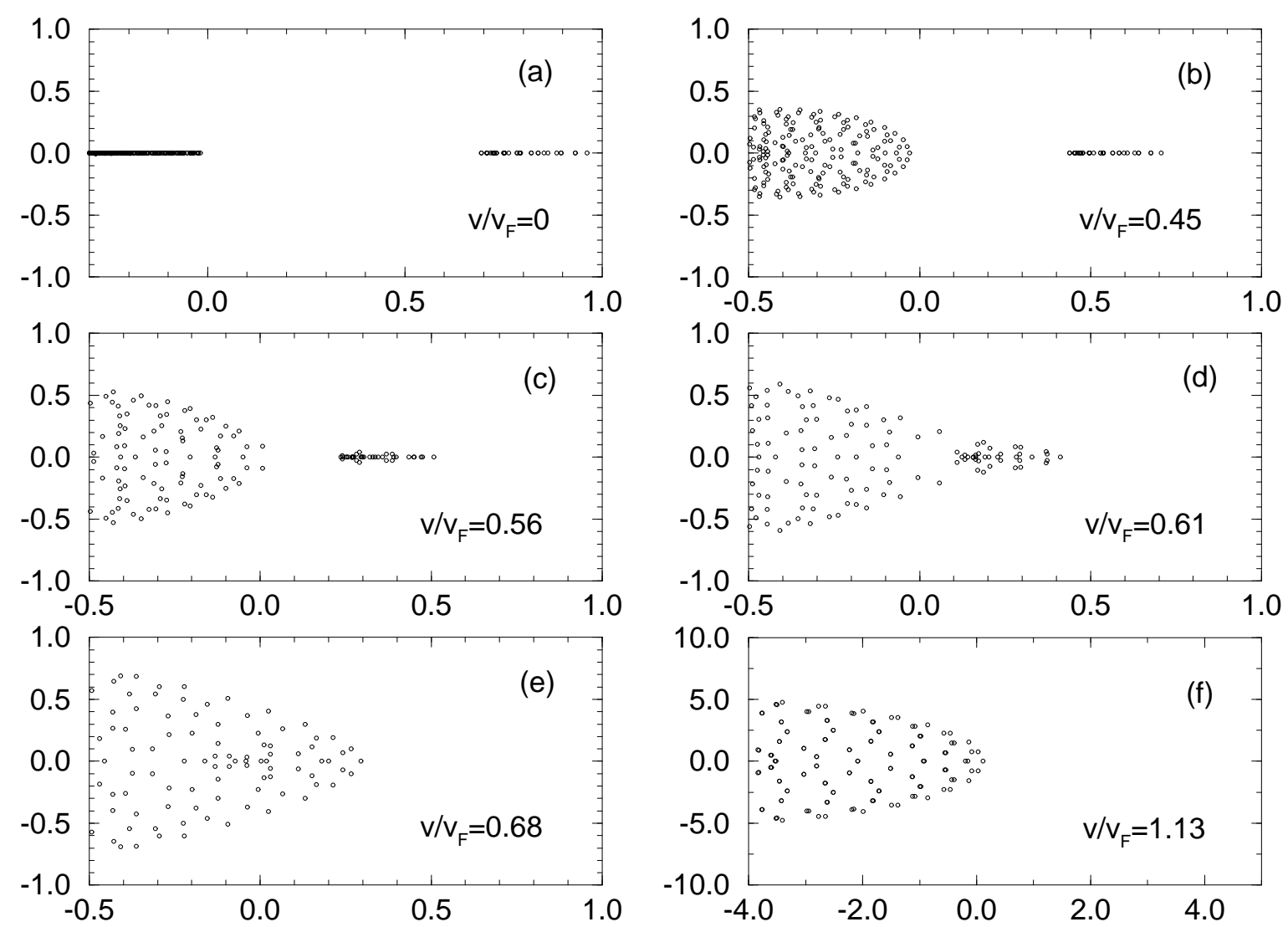

FIG. 6. Complex nonhermitian eigenvalue spectra at six different drift velocities for a two dimensional system with a hexagonal oasis (with growth rate $a=29.5 w^{\prime}$, where $w^{\prime}=1$ and $w^{\prime} \equiv 2 w / 3$ ) in a desert (with negative growth rate $-\epsilon a=-0.5 w^{\prime}$ ) with periodic boundary conditions. The growth rates of the spectra are divided by the difference of the growth rates inside and outside the oasis $U_{0} \equiv a+\epsilon a=30 w^{\prime}$. The drift velocities are directed along the $30^{\circ}$ axis of the triangular unit cell. Their respective values (divided by the Fisher wave velocity inside the oasis $v_{F}=2 \sqrt{a D}$ ) are indicated next to each figure. The spectra are extracted from numerical simulations of the triangular lattice model with 400 sites, with a hexagonal oasis with 37 sites, and diffusion constant $D=1.5 w^{\prime} \ell_{0}^{2}$, with $\ell_{0}=1$. (This figure actually only shows the part of the spectrum which gives a good approximation to the continuum problem.)

At zero velocity all eigenvalues are real (figure 6(a)). Just as in one dimension, with increasing convection velocity, the real growth rates of the localized states decrease while the mobility edge (i.e. the rightmost eigenvalue of the parabolic envelope of the delocalized states with complex eigenvalues) remains unchanged (b). Unlike in the one dimensional case, there are delocalized states with similar real parts of their eigenvalues, but a spread in the imaginary parts. (The reason is that in two and higher dimensions there are delocalized states that can carry a quantum mechanical current in directions other than the convection velocity 12,15.) Also, as the convection velocity is increased, some of the localized states become delocalized (signaled by eigenvalues which escape into the complex 
plane [12]) even before joining the parabola of delocalized states (c). The parabola formed by the eigenvalues of the delocalized states widens as the imaginary parts of the eigenvalues grow with the convection velocity, just as we saw for one dimensional systems. For even higher convection velocity the eigenvalues of the localized states enter the parabola of the delocalized states. The mobility edge is ill defined at these intermediate velocities (d). As the convection velocity $v$ approaches the Fisher wave velocity $v_{F}=2 \sqrt{a D}$, the upper edge of the band settles again into its fixed value (approximately $\operatorname{Re}\{\Gamma\}=0$ for the given parameter values), where it remains for all higher velocities (f).

Dynamical properties of the full nonlinear system can be studied by discretizing Eq. (3.2) also in time and using Runge Kutta methods to solve the equation numerically [16, 17, 14, 18]. Although we hope to present a more thorough study at a later date, Fig. 7 shows preliminary results in the form of snapshots of a bacterial colony drifting and expanding in a 2 dimensional system on a triangular mesh, with convection and quenched random growth rates. The initial condition for the system shown was a delta function like spatial distribution concentrated in the upper right hand corner. Theoretical predictions in the limit of high convection velocity for the long time scaling behavior of a growing and drifting droplet of bacteria in the linearized regime were given in [8].

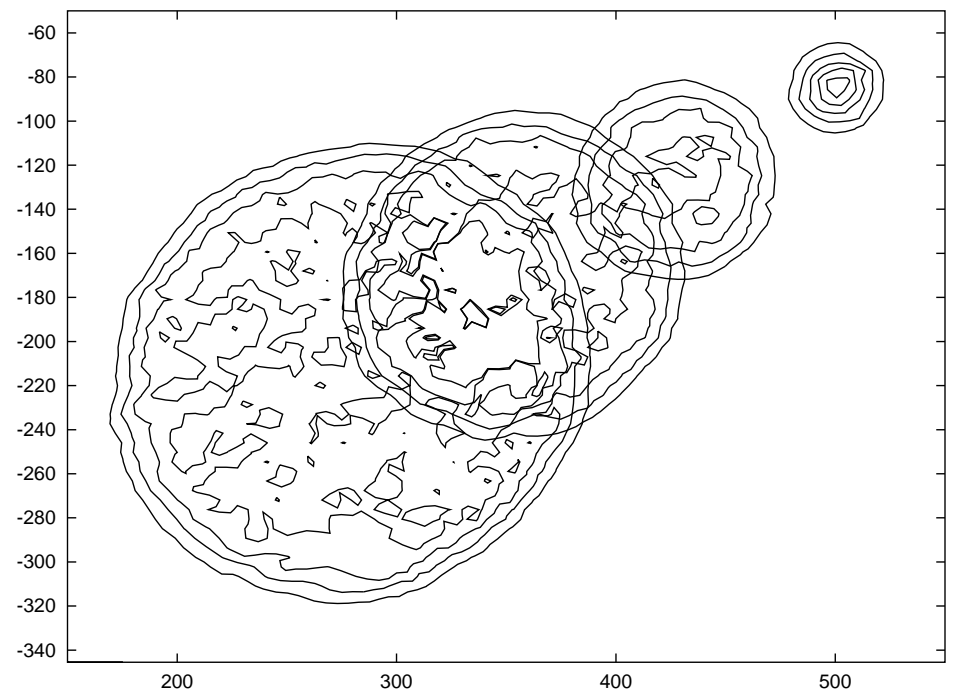

FIG. 7. Contour plots for snapshots of the distribution of bacteria in a two dimensional landscape with (quenched) random growth rates, obtained from the full nonlinear problem for convection in the direction relative to a triangular lattice shown in Fig. 4, with velocity parameter $g=1, w^{\prime} \equiv 2 w / 3=1, b=1$, and $\ell_{0}=1$. The random growth rates $U(\mathbf{x})$ are uniformly distributed with values ranging from $-0.3 w^{\prime}$ to $0.7 w^{\prime}$. At time $\mathrm{t}=0$ the bacterial population was zero everywhere, except at one lattice point where it was set to 1 . The snapshots show the expanding and drifting droplet after 250, 500, 750, and 1000 time steps of size $\delta t=0.1 / w^{\prime}$. The contour lines marking population density $c=0.1$ are the innermost contour for the snapshot after 250 time steps, the second to outermost contour for the snapshots after 500 and 750 time steps and the outermost contour for the snapshot after 1000 time steps. The simulated system had $500 \times 500$ sites.

\section{EXACT RESULTS FOR LATTICE MODELS IN ONE DIMENSION}

Consider the lattice models discussed in the previous section with a random growth rate $U(\mathbf{x})$ chosen from an identical proability distribution at every lattice site $\mathbf{x}$. If the drift parameter $\mathbf{g} \propto \mathbf{v}$ in Eq. (3.3) is set to zero, the growth operator $\tilde{\mathcal{L}}$ becomes formally identical to a Hermitian Hamiltonian describing a quantum mechanical particle which hops between lattice sites in a disordered medium. A number of exact results are known for this problem in one dimension; we review here recent exact results for the non-Hermitian generalization embodied in Eq. (3.3) [19, 21], with an emphasis on the winding numbers and complex currents associated with extended states 111 .

A numerical study of the eigenvalue spectrum of the lattice model in one dimension 12 reveals the following: when $g=0$, all eigenfunctions $\psi_{n}(x)$ are localized with a bounded point-like spectrum $\left\{\Gamma_{n}\right\}$ of eigenvalues lying on the real axis, similar to the subset of localized states which fall to the right of the mobility edge for the square well spectrum shown in Fig.2. Localization of all states by a random potential is the expected result for quantum particles in a 
disordered medium in one dimension. The situation changes dramatically, however, when $g \neq 0$. For $g$ greater than a critical value $g=g_{c}$, a bubble of complex eigenvalues appears in the center of the band, similar to the response to convection for arbitrary $g$ of the continuous spectrum part of the spectrum for a square well shown in Fig.2 The eigenfunctions associated with this bubble are extended [12. Eigenvalues outside this bubble remain real, and are related by a simple rigid band shift to their values for $g=0$. The right and left eigenfunctions $\phi_{n}^{R, L}(x)$ for states outside the bubble remain localized, and are related to the eigenfunctions $\psi_{n}\left(x_{j}\right)$ of the Hermitian problem for $g=0$ by

$$
\phi_{n}^{R, L}\left(x_{j}\right)=e^{ \pm g x_{j}} \psi_{n}\left(x_{j}\right)
$$

We assume here a one dimensional lattice with unit spacing $\ell_{0}=1$.

We shall focus on the eigenfunctions and complex currents associated with the band of extended states in one dimension for lattice growth models with disorder in one dimension [11]. Unlike delocalized states in Hermitian disordered systems (where the eigenfunctions can always be chosen to be real), we show that these complex eigenfunctions are characterized by a conserved winding number $n$, even when the disorder is strong. Such topological quantum numbers can be used to label the eigenvalue spectrum $\Gamma_{n}(g)$, where $g$ is the asymmetry parameter. A study of the eigenvalue trajectories as a function of $g$ then leads to complex currents defined by $J_{n}=-i \frac{\partial \Gamma_{n}}{\partial g}$, which determine the response of the $n$-th eigenvalue to changes in the drift velocity.

The matrix representation of the one dimensional non-Hermitian Liouville operator (3.3) in a basis of $N$ sites localized at positions $\left\{x_{j}, j=1, \cdots, N\right\}$ reads,

$$
\tilde{\mathcal{L}}=\left(\begin{array}{ccccc}
U_{1} & \frac{w_{1}}{2} e^{g_{1}} & 0 & . . & \frac{w_{N}}{2} e^{-g_{N}} \\
\frac{w_{1}}{2} e^{-g_{1}} & U_{2} & \frac{w_{2}}{2} e^{g_{2}} & . . & 0 \\
0 & \frac{w_{2}}{2} e^{-g_{2}} & U_{3} & . . & 0 \\
: & : & : & : & : \\
\frac{w_{N}}{2} e^{g_{N}} & 0 & 0 & . . & U_{N}
\end{array}\right) .
$$

where we assume periodic boundary conditions, and the constant background of the growth rate $A$ has been omitted.

The matrix displayed in Eq. (4.2) (tridiagonal, with nonzero corner matrix elements) is actually a generalization of Eq. (3.3): As before, $U_{j}$ is a zero mean random growth rate chosen independently for each site. However, the hopping rate between sites $i$ and $i+1, w_{i}$, is now a random function of position, which allows for fluctuations in the local diffusion constant. We have also allowed for fluctuations in the local convection velocity $g_{i}$. This latter variation can be eliminated via similarity transformation which preserves the eigenvalues of $\tilde{\mathcal{L}}$, and produces only a smooth, nonsingular change in the eigenfunctions. It is helpful to carry out this transformation in two stages. The first isolates all effects of convection on the bond connecting the $N$ th and 1st site: Upon defining

$$
S_{1}=\left(\begin{array}{ccccc}
1 & 0 & 0 & . . & 0 \\
0 & e^{-g_{1}} & 0 & . . & 0 \\
0 & 0 & e^{-g_{1}-g_{2}} & . . & 0 \\
: & : & : & : & \vdots \\
0 & 0 & 0 & . . & e^{-\sum_{j=1}^{N-1} g_{j}}
\end{array}\right)
$$

we have

$$
\mathcal{L}^{\prime}=S_{1}^{-1} \tilde{\mathcal{L}} S_{1}=\left(\begin{array}{ccccc}
U_{1} & \frac{w_{1}}{2} & 0 & . . & \frac{w_{N}}{2} e^{-\sum_{j=1}^{N} g_{j}} \\
\frac{w_{1}}{2} & U_{2} & \frac{w_{2}}{2} & . . & 0 \\
0 & \frac{w_{2}}{2} & U_{3} & . . & 0 \\
: & : & : & : & : \\
\frac{w_{N}}{2} e^{\sum_{j=1}^{N} g_{j}} & 0 & 0 & . . & U_{N}
\end{array}\right) .
$$

Except for the corner matrix elements, $\mathcal{L}^{\prime}$ describes Hermitian population growth without convection. A second similarity transformation,

$$
S_{2}=\left(\begin{array}{ccccc}
1 & 0 & 0 & . . & 0 \\
0 & e^{g} & 0 & . . & 0 \\
0 & 0 & e^{2 g} & . . & 0 \\
: & : & : & : & : \\
0 & 0 & 0 & . . & e^{(N-1) g}
\end{array}\right)
$$


where $g=\frac{1}{N} \sum_{j=1}^{N}$ then redistributes the convection uniformly on all bonds,

$$
\mathcal{L}=S_{2}^{-1} \mathcal{L}^{\prime} S_{2}=S_{2}^{-1} S_{1}^{-1} \tilde{\mathcal{L}} S_{1} S_{2}=\left(\begin{array}{ccccc}
U_{1} & \frac{w_{1}}{2} e^{g} & 0 & . . & \frac{w_{N}}{2} e^{-g} \\
\frac{w_{1}}{2} e^{-g} & U_{2} & \frac{w_{2}}{2} e^{g} & . . & 0 \\
0 & \frac{w_{2}}{2} e^{-g} & U_{3} & . . & 0 \\
: & : & : & : & : \\
\frac{w_{N}}{2} e^{g} & 0 & 0 & . . & U_{N}
\end{array}\right)
$$

The composite similarity matrix

$$
S_{i j}=\left(S_{1} S_{2}\right)_{i j}=\delta_{i j} \exp \left[-\sum_{k=1}^{j-1} g_{k}+\frac{(j-1)}{N} \sum_{k=1}^{N} g_{k}\right]
$$

thus leads to a population growth problem whose eigenvalues reflect a mean convection parameter $g=\frac{1}{N} \sum_{j=1}^{N} g_{j}$. For the $g_{j}$ 's chosen independently at each site, the sample to sample fluctuations should fall off like $\sigma / N$ where $\sigma$ is the variance of the distribution. As $N$ grows one may thus replace the fluctuating quantity $g_{i}$ by a disorder independent average value $g$, as we shall do in the rest of this paper. This result implies universality in the response to random convection in one dimensional growth models - nonuniform convection velocities may be mapped into a uniform average velocity via a similarity transformation, provided one applies the transformation (4.7) to the eigenfunctions as well.

For a 1D ring with random parameters $\left\{U_{i}\right\},\left\{w_{i}\right\}$ and $g=0$, all the eigenfunctions of (4.4) are real and localized, and its eigenvalues are real and discrete 12,8]. We assume for simplicity that all $w_{i}>0$ and that the chain is large but finite, such that although the spectrum is discrete, the length of the chain is much larger than the maximal localization length of an eigenmode.

In a typical one-dimensional disordered system with $g=0$, the localization length $\xi$ (defined via the exponential fall off of the eigenfunction) is larger at the center of the band and smaller at the tails. The criterion for delocalization of the asymmetric system via convection (see Eq. (4.1)) is $\kappa \ell_{0}<g$, where $\kappa \equiv 1 / \xi[12]$. As a result, pairs of complex energies representing delocalized states first appear as a "bubble" at the center of the band, which then spreads into the band tails as the convection parameter $g$ is increased (see Fig. 9). To study the complex currents associated with delocalized states we follow Refs. [19 21] and exploit the relation between the complex spectrum of the asymmetric problem with $g \neq 0$ and the real eigenvalues of a "background" localized problem with $g=0$, with the same realization of disorder.

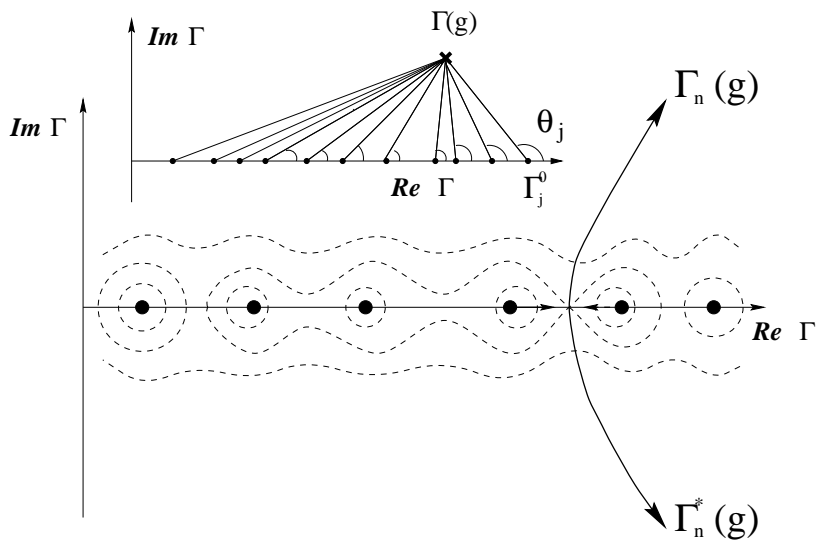

FIG. 8. Eigenvalue trajectories in the complex plane. Points on the real axis are eigenvalues for the Hermitian problem for $g=0$. The complex eigenvalue trajectories $\Gamma_{n}(g)$ and $\Gamma_{n}^{*}(g)$ are orthogonal to the level curves (dashed lines) defined by Eq.4.10). Inset: Angles entering Eq.44.9) for a given eigenvalue $\Gamma(g)$.

The condition for a complex number $\Gamma=\Gamma_{R}+i \Gamma_{I}$ to be an eigenvalue of the matrix $\mathcal{L}(g)$ displayed in Eq. (4.6) is $\operatorname{Det}[\Gamma I-\mathcal{L}(g)]=0$, where $I$ is the identity matrix. Upon expanding in cofactors, we find that $\Gamma$ is an eigenvalue provided [19,22]

$$
\operatorname{Det}[\Gamma I-\mathcal{L}(g=0)]=\prod_{i=1}^{N}\left(\Gamma-\Gamma_{i}^{0}\right)
$$




$$
=2[\cosh (g N)-1] \prod_{j=1}^{N}\left(\frac{w_{j}}{2}\right)
$$

where the $\left\{\Gamma_{i}^{0}\right\}$ are the (real) eigenvalues of the background matrix $\mathcal{L}(g=0)$. To extract winding numbers, we first observe that the right hand side of Eq. (4.8) is real and positive. As a result, the phases of the complex numbers in the left product of (4.8) for each complex $\Gamma$ should sum up to (see inset to Fig. 8)

$$
\sum_{i} \cot ^{-1}\left(\frac{\Gamma_{R}-\Gamma_{i}^{0}}{\Gamma_{I}}\right)=p \pi
$$

with $p=2 n$, where $n$ is an integer, and the function $\cot ^{-1}(x)$ varies from $\pi$ to 0 as $x$ goes between $-\infty$ and $\infty$.

As $\Gamma_{I} \rightarrow 0$, each term in Eq.(4.9) gives $\pi$ for every eigenvalue $\Gamma_{i}^{0}$ to the right of $\Gamma_{R}$, and zero for each eigenvalue to the left (see Fig.8). To satisfy (4.9), the eigenvalue must leave the real axis and enter the complex plane at the gap between the $2 \mathrm{n}$-th and the $2 \mathrm{n}+1$-th eigenvalues of the $g=0$ "background" system. We call $n$ the index of the trajectory $\Gamma_{R}(g)+i \Gamma_{I}(g)$ in the complex plane.

We see immediately from (4.9) that the rightmost eigenvalue (with $n=0$ ) must remain real, consistent with Perron-Frobenius theorem 23]. The corresponding nodeless eigenfunction corresponds to the ground state of $-\mathcal{L}^{\prime}$. For $N$ even, particle-hole symmetry implies that both the rightmost and leftmost eigenvalues are always real. More generally, for a fixed value of $n$, the set of all $\left[\Gamma_{R}(g), \Gamma_{I}(g)\right]$ satisfying Eq. (4.9) defines a curve in the complex plane, as illustrated in Fig. 8 . Henceforth, we assume $N$ even for simplicity.

A more complete description of the eigenvalue trajectory results from taking the logarithm of the modulus of Eq. (4.8). In the limit $N g>>1$ one finds a second constraint on $\Gamma(g)$, namely, 19 21]

$$
|g|=-\ln \left(\frac{w}{2}\right)+\frac{1}{N} \sum_{i} \ln \left(\left|\Gamma-\Gamma_{i}^{0}\right|\right)
$$

where $\left|\Gamma-\Gamma_{i}^{0}\right|=\sqrt{\left(\Gamma_{R}-\Gamma_{i}^{0}\right)^{2}+\Gamma_{I}^{2}}$ and $w=2\left[\prod_{i=1}^{N} \frac{w_{j}}{2}\right]^{\frac{1}{N}}$.

This constraint is described graphically in Fig. 8, which shows schematically the level curves defined by Eq. (4.10) near the band center for three values of $g$. These are lines of constant potential for an equivalent $2 \mathrm{~d}$ electrostatic problem with charges at the positions of the localized point spectrum for $g=0$. When $g$ is small, the constraint is solved by eigenvalue pairs on the real axis in the gaps between neighboring $\Gamma_{j}^{0}$ 's indexed by $p=2 n$. As $g$ increases, successive pairs of eigenvalues eventually merge at a saddle point in the potential contours and detach from the real axis at right angle. For a given real energy $\Gamma_{R}$, Thouless has defined an energy dependent inverse localization length $\kappa\left(\Gamma_{R}\right)$ for the associated $g=0$ Hermitian problem [22],

$$
\kappa\left(\Gamma_{R}\right)=-\ln \left(\frac{w}{2}\right)+\frac{1}{N} \sum_{i} \ln \left(\left|\Gamma_{R}-\Gamma_{i}^{0}\right|\right) .
$$

Upon comparing with Eq. (4.10), we see that $\Gamma_{I}$ becomes nonzero whenever $|g|>\kappa\left(\Gamma_{R}^{\prime}\right)$ where $\left(\Gamma_{R}^{\prime}, 0\right)$ is the detachment point of the eigenvalue pair.

As $g$ increases above $\kappa\left(\Gamma^{\prime}\right)$, Eqs. (4.9) and (4.10) define a unique pair of complex eigenvalue trajectories $\Gamma_{n}(g)$ and $\Gamma_{n}^{*}(g)$ for every value of $n$. Upon passing to the limit $N \rightarrow \infty$, the spectrum $\left\{\Gamma_{j}^{0}\right\}$ for $g=0$ closes up, and is described by a density of states $\rho_{0}(\lambda)$. Eqs. (4.9) and (4.10) may then be recombined into a single complex equation, namely

$$
\int_{-\infty}^{\infty} d \lambda \rho_{0}(\lambda) \ln \left[\Gamma_{n}(g)-\lambda\right]=\ln \left(\frac{w}{2} e^{|g|}\right)+i \pi\left(\frac{2 n}{N}\right) .
$$

where

$$
\ln [\Gamma(g)-\lambda]=\ln \left[\left|\Gamma_{R}+i \Gamma_{I}-\lambda\right|\right]+i \cot ^{-1}\left[\left(\Gamma_{R}-\lambda\right) / \Gamma_{I}\right] .
$$

For simplicity we set the average growth rate to zero. Then in the limit $N>>1$ and for a density of states function symmetric around the special detachment point with $\Gamma_{R}^{\prime}=0$, there is a purely imaginary trajectory of the form $\Gamma(g)=i \Gamma_{I}(g)$ with $n=N / 2$. For fixed $g$, Eq. (4.12) thus leads to an implicit formula for the "height" $\Gamma_{I}^{\max }$ of the bubble of complex eigenvalues in the center of the band (see Fig. 9), namely,

$$
\frac{1}{2} \int d \lambda \rho(\lambda) \ln \left[\lambda^{2}+\left(\Gamma_{I}^{\max }\right)^{2}\right]=|g|+\ln \left(\frac{w}{2}\right) .
$$


This integral vanishes, as expected [20], in the "one way" limit, $g \rightarrow \infty$ with $\frac{w}{2} e^{|g|}=1$. For other detachment points, the eigenvalue trajectories curve to the left or right as required by the constraint of Eq. (4.10) (see Fig. 8).

The analysis above suggests that the imaginary parts of all $\left\{\Gamma_{n}(g)\right\}$ (except the two which remain on the real axis), diverge as $|g| \rightarrow \infty$. It is then expected that all eigenfunctions $\phi_{n}(j)$ are approximately plane waves, $\phi_{n}(j) \sim$ $\exp \left(i k_{n} r_{j} / \ell_{0}\right)$, with free particle eigenvalue energies $\Gamma_{n}(g)=w \cos \left(k_{n}+i g\right)$ [8,12]. For large $\left|\Gamma_{I}(g)\right|$, Eq. (4.12) leads to

$$
\Gamma_{n}(g) \approx \frac{w}{2} \exp (|g|+2 i \pi n / N)
$$

Comparison with the free particle spectrum at large $|g|$ shows immediately that the index $n$ of the eigenvalue trajectory and the wave vector are related, $k_{n}=2 \pi n / N$. These wave eigenfunctions spiral around the origin in the complex plane as one moves along the 1d lattice of the tight binding model sites, leading to a well defined winding number $n$. The winding number associated with the eigenvalue $\Gamma_{n}^{*}(g)$ in the lower half plane is then $-n$.

As $g$ decreases, it can be shown that the associated delocalized wave function $\phi_{n}(j ; g)$ must remain nonzero for all $j$, so long as the eigenvalue retains an imaginary part [24. Thus, the winding number must be preserved as $g$ decreases, i.e., the winding number is a topological invariant along an eigenvalue trajectory. The projection of such a delocalized eigenfunction is illustrated in the inset to Fig. 9 .

As $N \rightarrow \infty$, we can replace the winding index $n$ by the continuous variable $k_{n}=\frac{2 \pi}{N} n$. Eq. (4.12) then shows quite generally that the complex spectrum is a function only of the combination $g+i k$, i.e., $\Gamma_{n}(g)=\Gamma(g+i k)$. It then follows from the Cauchy-Riemann relations that all eigenvalue trajectories are at right angles to the lines of constant g, i. e. $\frac{d \Gamma(g)}{d g}=-i \frac{d \Gamma(g)}{d k}$.

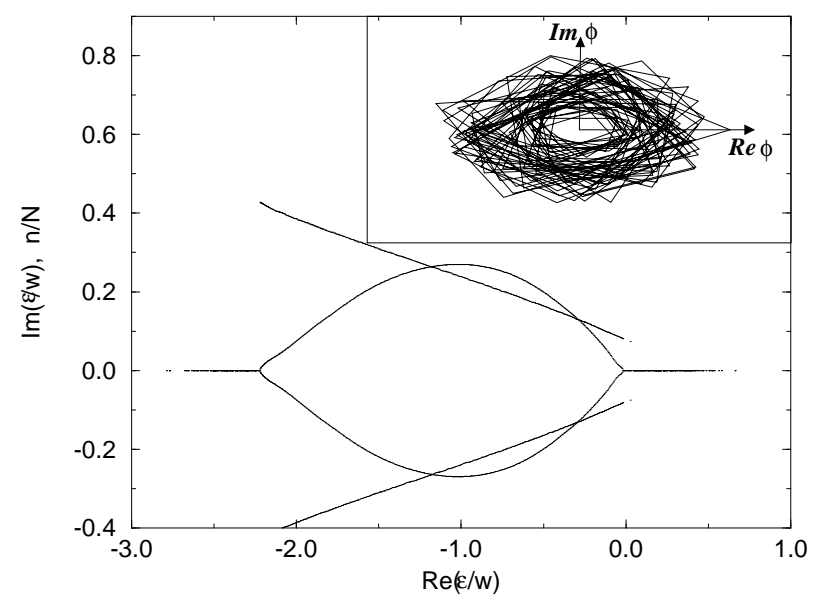

FIG. 9. Spectrum and winding numbers for $N=1000$ with $U_{j}$ uniformly distributed in the interval $[-1,1]$, and $g=0.4$. Inset: Projection onto the complex plane of an extended eigenfunction near the center of the bubble with $N=200, g=1$, and winding number $n=100$.

An explicit formula for the complex current results, moreover, from differentiating Eq. (4.12) with respect to $g$,

$$
\begin{aligned}
J\left[\Gamma_{n}(g)\right] & =-i \frac{\partial \Gamma_{n}(g)}{\partial g} \\
& =\left[i \int d \lambda \frac{\rho_{0}(\lambda)}{\Gamma_{n}(g)-\lambda}\right]^{-1} \equiv-i G_{0}^{-1}\left[\Gamma_{n}(g)\right]
\end{aligned}
$$

Evidently, the current associated with a particular complex eigenvalue $\Gamma_{n}(g)$ is determined by the Green's function of the $g=0$ problem, evaluated at the complex energy $\Gamma_{n}(g)$.

Fig. 9 shows a typical eigenvalue spectrum for $\mathcal{L}$ in one dimension superimposed on the winding numbers associated with the extended eigenfunctions. The inset illustrates how the winding number is defined for a typical extended state. The winding numbers are exactly $\pm N / 2$ at the band center, and their magnitudes decrease monotonically as one moves toward the upper edge of the band, i.e., toward the lowest energies of the corresponding Hamiltonian. 
The winding numbers remain finite up to the mobility edge separating complex and real eigenvalues, but become undefined in the band tails, where all wave functions are real. At the transition, an overall constant phase factor can be adjusted so that the imaginary part of the eigenvector vanishes while its real part remains finite. In this case, the winding index classification can be replaced by counting the nodes of the real, localized wave function [22].

\section{PSEUDOSPECTRA AND SENSITIVITY TO BOUNDARY CONDITIONS}

In this review, we have explored the spectral properties of the linearized growth operator $\mathcal{L}$ in order to learn about the time evolution of the growing populations using spectral decomposition, as in Eq. (2.5). In the recent years, however, it has been recognized that such a straightforward analysis of non-Hermitian operators and matrices (such as the Liouville operator in Eq. (2.3)) can in some circumstances lead to inaccurate predictions [25 27]. In this section we discuss convection-diffusion with a random growth rate in this context. We argue that, while some non-Hermitian problems are indeed abnormally sensitive to small perturbations, our results (provided the boundary conditions admit a net population flux) are quite robust. We consider a linear, (possibly non-Hermitian), operator which admits matrix representation in a Hilbert space. When convenient, we interchange freely between the matrix and the operator descriptions.

Consider first the Hermitian operator, $\mathcal{H}$, which has a complete set of orthogonal eigenvectors. If the spectrum of $\mathcal{H}$ is non-degenerate, any eigenvalue $\Gamma_{n}$ corresponds uniquely to an eigenstate. When a two-fold degeneracy occurs, there are two independent eigenvectors corresponding to the same eigenvalue, and one may orthogonalize these states using the Graham-Schmidt scheme [23]. If $\mathcal{H}$ is an evolution operator, its response to external perturbation with "frequency" $z$ is determined by the resolvent (i.e. the Green's function) $(z I-\mathcal{H})^{-1}$. The resolvent norm [28] diverges if $z$ is a spectral point of $\mathcal{H}$. For small deviations, i.e., $z=\Gamma_{n}+\delta z$, for some $\Gamma_{n}$ one may use the diagonalized representation of the resolvent to see that its norm is proportional to $(\delta z)^{-1}$.

For a non-Hermitian operator, or, in particular, a real asymmetric object, such as $\mathcal{L}(g)$, the situation is different. If the spectrum of this non-normal operator is non-degenerate, it admits two complete, biorthogonal states of left and right eigenfunctions [29]. For any eigenvalue $\Gamma_{n}(g)$ the left eigenstate $<\phi_{L}^{n} \mid$ satisfies $<\phi_{L}^{n}\left|\mathcal{L}(g)=\Gamma_{n}(g)<\phi_{L}^{n}\right|$ while the right state satisfies $\mathcal{L}(g)\left|\phi_{R}^{n}>=\Gamma_{n}(g)\right| \phi_{R}^{n}>$. The biorthogonality condition means that

$$
<\phi_{L}^{m} \mid \phi_{R}^{n}>=\delta_{m, n}
$$

Unlike the Hermitian case, the left and right eigenstates are not simple complex conjugates. Hence, the normalization condition (5.1) implies no restriction on the usual Hilbert norm of the left and right eigenfunction considered separately, and the only normalized quantity is the "overlap integral" between them. In the following we explore the significance of this observation.

Non-normality also implies that, in the generic case, as two eigenstates coincide (when the spectrum is "degenerate") the corresponding eigenvectors also coincide. The subspace spanned by these vectors is one dimensional, and an $N \times N$ matrix is defective, i.e., it fails to span the $N$ dimensional vector space associated with it. An illustration of the "almost degenerate" situation for the left and right eigenvectors in a $2 \times 2$ space is represented in Fig. 10. Note that $<L_{2} \mid$ is orthogonal to $\mid R_{1}>$, and $<L_{1} \mid$ is orthogonal to $\mid R_{2}>$ as required by Eq. (5.1). 


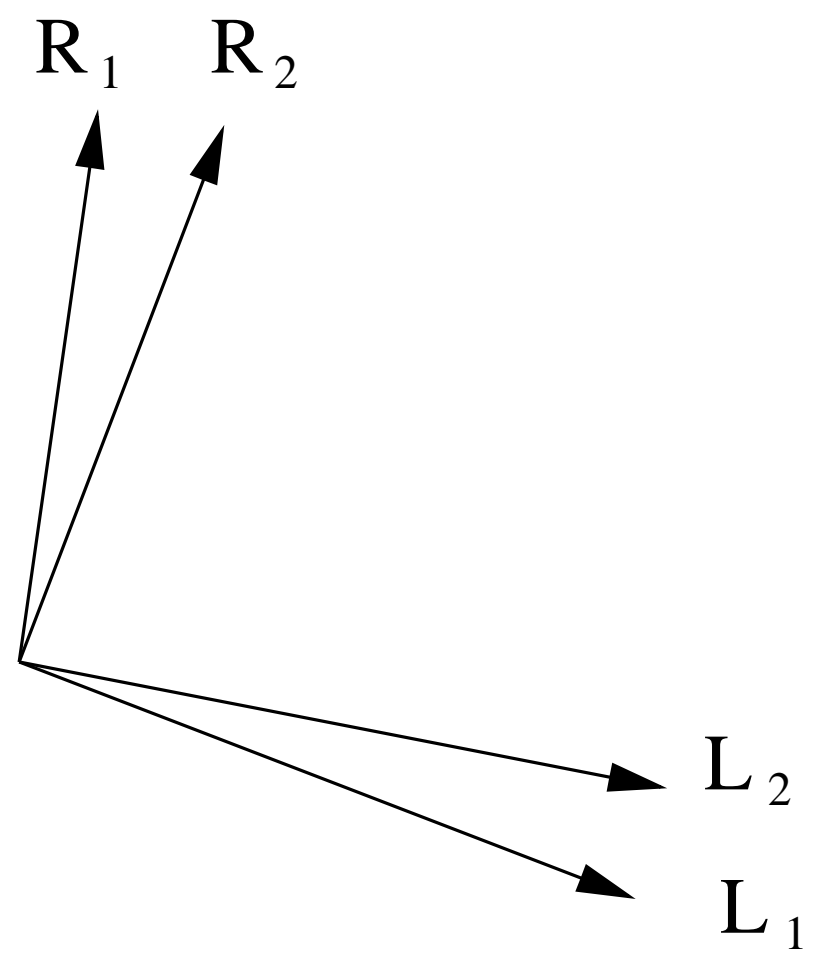

FIG. 10. Two pairs of almost degenerate, left and right eigenvectors of a non-normal matrix. The condition (5.1) implies that the $R_{1}$ and $L_{2}$ are orthogonal, as well as $R_{2}$ and $L_{1}$. Close to degeneracy $L_{1}$ and $L_{2}$ almost coincide, hence $R_{1}$ is "almost orthogonal" to $L_{1}$. As illustrated in Fig. 11, a typical value of the poperly normalized eigenfunctions must then be very large in order to satisfy the normalization condition $\left\langle L_{n} \mid R_{n}\right\rangle=1$.

The notion of pseudospectra has been introduced [25] in order to quantify the problems which may arise when spectral analysis is applied to non-normal operators. While the spectrum $\Gamma(\mathcal{L})$ of the operator $\mathcal{L}$ is defined as the set of points (in the complex plane) which are eigenvalues of $\mathcal{L}$, the $\epsilon$-pseudospectrum $\Gamma_{\epsilon}(\mathcal{L})$ is defined as a set of points which imitate a true eigenvalue to within a small parameter $\epsilon>0$. Formally, the definition is:

$$
\Gamma_{\epsilon}(\mathcal{L})=\left\{\text { complex numbers } z \text { such that }\left\|(z I-\mathcal{L})^{-1}\right\| \geq \epsilon^{-1}\right\}
$$

(note that if $z$ is a spectral point, $(\|z I-\mathcal{L}\|)^{-1}=\infty$ ). Eq. (5.2) implies that the response of the system to a small time dependent perturbation with "frequency" $z$ mimics a resonance up to times of order $t \sim 1 / \epsilon$. If the pseudospectral region is large for small $\epsilon$, the system is highly unstable against weak external perturbations even far away from the resonance.

Another definition (mathematically equivalent to (5.2)) is,

$$
\begin{aligned}
\Gamma_{\epsilon}(\mathcal{L})= & \{\text { complex numbers } z \text { which belong to the spectrum of } \mathcal{L}+\delta \mathcal{L}, \\
& \text { for all } \delta \mathcal{L} \text { such that }\|\delta \mathcal{L}\| \leq \epsilon\}
\end{aligned}
$$

Eq. (5.3) defines the pseudospectrum via the sensitivity of $\mathcal{L}$ to small perturbations in the growth operator. Again, broad pseudospectral regions for small $\epsilon$ imply high sensitivity to small perturbations. 


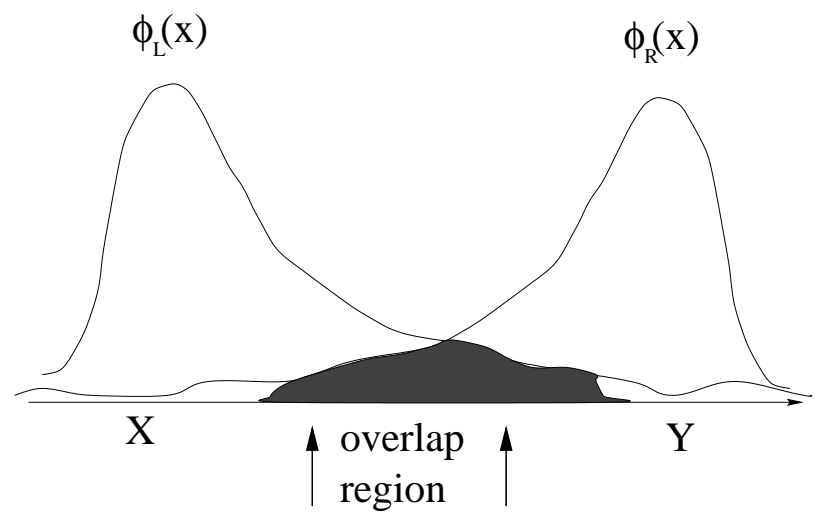

FIG. 11. Sketch of the right and left eigenfunctions of a non-normal operator. Since the normalization is given by the overlap (shaded) region, the spectrum of such a system is very unstable against a non-diagonal perturbation which connects the regions $X$ and $Y$.

The reason for such an extreme sensitivity in the context of the random growth operator considered here, can be clarified if one looks at the (localized) left and right states shown in Fig. 11. For simplicity, we show the "ground state" (most rapidly growing) left and right eigenfunctions, but similar considerations apply to excited states as well. For nonzero convection velocities the peaks in the eigenfunction will be shifted away from the overlap region according to Eq. (4.1). If the overlap integral defined in Eq. (5.1) is small, the amplitude after normalization of both $\phi_{L}^{n}$ on the left side of Fig. 11 and of $\phi_{R}^{n}$ at the right side of Fig. 11 will be very large. Perturbation theory will then yield huge corrections to the eigenvalues if $\delta L$ has an off diagonal matrix element $\left\langle\phi_{L}|\delta \mathcal{L}| \phi_{R}>\right.$ connecting regions $X$ and $Y$. The pseudospectral instability thus becomes stronger and stronger as the overlap integral $<\phi_{L}^{m} \mid \phi_{R}^{m}>$ becomes small compared with the norm of the individual eigenvectors.

Fig. 10 illustrates why this overlap integral also becomes smaller as eigenvalues approach degeneracy. According to the biorthogonality condition (5.1), if $n \neq m$, the right eigenvector $\phi_{R}^{m}$ should be orthogonal to all the left states $\phi_{L}^{n}$. Near the degeneracy point, when the two left eigenvectors almost coincide, the right eigenvectors become nearly orthogonal to their own left counterparts, and the overlap integral almost vanishes. Thus, nearly degenerate eigenvalues in a non-Hermitian operator can cause extreme sensitivity to small off-diagonal perturbations, corresponding to large pseudospectral regions for small $\epsilon$. The same mechanism may have caused the unstable spectra of large non-Hermitian matrices (with random entries connecting sites arbitrarily far apart) observed recently by Chalker and Mehlig [30].

With this background in mind, let us consider the pseudospectral properties of the convection-diffusion operator with random growth considered in this review. The pseudospectrum of the convection-diffusion operator

$$
\mathcal{L}_{c d}=\partial_{x}^{2}+v \partial_{x}
$$

has been considered by Reddy and Trefethen [27], for "absorbing" boundary conditions. In our language this situation corresponds to a uniform growth rate with no population allowed outside of the $[0, d]$ region. As for an infinite potential well in quantum mechanics, the eigenfunctions satisfy $\phi(0)=\phi(d)=0$. For $v=0, \mathcal{L}_{c d}$ is Hermitian with a complete set of orthogonal eigenfunctions, indexed by an integer $m, \phi_{m} \sim \sin (m \pi x / d)$ with the corresponding eigenvalues $\Gamma_{m}=-\pi^{2} m^{2} / d^{2}$. As $v$ is increased, the transformation (4.1) gives left and right eigenfunctions of the form $\phi_{m}^{L, R} \sim \exp ( \pm v x) \sin (m \pi x / d)$, leading to peaks near the boundaries, similar to those sketched in Fig. 11. The spectrum undergoes a rigid shift of $-v^{2} / 4$ for all $m$, since the above transformation always works for these boundary conditions. The result of this left-right asymmetry is, as discussed above, a broad pseudospectral region. In fact, as shown in Ref. [27] (for the case $v=1$ ), all points in the complex plane contained inside the parabola

$$
\operatorname{Re} z=-(\operatorname{Im} z)^{2}
$$

are contained in $\Gamma_{\epsilon}\left(\mathcal{L}_{c d}\right)$ for small $\epsilon$, and the norm in Eq. (5.2) diverges exponentially as $d \rightarrow \infty$. In the light of our discussion above - this extreme instability arises from small perturbations of the evolution operator which connect the two bumps at the edges, i.e., from small changes of the boundary conditions.

We show that the results in this review are in fact unaffected by this interesting instability. First, we note that for periodic boundary conditions, the eigenstates of the non-Hermitian operator $\mathcal{L}_{c d}\left(\right.$ see Eq. (5.4)) are simply $\phi_{n}^{R, L}=$ $\exp \left( \pm i k_{n} x\right)$ with $k_{n}=2 \pi n / d$ and eigenvalues $\Gamma_{n}=-k_{n}^{2}+i v k_{n}$, i.e., the eigenvalues lie on the parabolic limit of the pseudospectral region illustrated in Eq. (5.5) for $v=1$. Since the wave functions in this case do not have the asymmetric form implied by Eq. (4.1), we expect stability against small perturbations. 
Consider the random matrix model of Sec. IV], now with a change of a single $w_{j}$ to allow boundary conditions similar to those of Ref. [27. Specifically, we insert a factor $\eta$ in front of the corner matrix elements of Eq. (4.2),

$$
w_{N} \rightarrow \eta w_{N} .
$$

By tuning $\eta$ to zero, we break the chain and discard the periodic boundary conditions. Upon making the same transformation which led to Eq. (4.4), we see that all effects of convection vanish when $\eta=0$, so the eigenvalue spectrum remains real for an arbitrary array of convection velocities $g_{i}$, similar to the results for "absorbing" boundary conditions in the simple convection-diffusion problem in Ref. [27]. If $\eta$ is set to zero in Eq. (4.4), it is easy to show that a 'gauge' transformation like (4.1) relates the eigenfunctions to their $g=0$ values for all values of $g$, with a trivial shift of the real $g=0$ eigenvalue spectrum.

The results change dramatically, however, for any $\eta>0$, no matter how small. Indeed, it is clear from Eq. (4.4) that the eigenvalue spectrum for nonzero $\eta$ is similar to that for $\eta=1$, provided we calculate the spectrum with effective values of $g^{ \pm}$,

$$
g^{ \pm}=g \pm \ln (\eta) / N,
$$

where the + sign refers to the lower left corner matrix element and the - sign refers to the upper right. All effects of $\eta$ vanish in the continuum limit $N \rightarrow \infty$, and the eigenvalues approach a universal spectrum like that in Fig. 9, for all $\eta \neq 0$. It is straightforward to show, using the methods of Sec. IV], that the eigenfunctions are also unchanged in the large $N$ limit. We conclude that the results for periodic boundary conditions are stable to a change in boundary conditions in one dimension, provided the chain is not actually broken by setting $\eta \equiv 0$.

Our conclusions about the stability of the spectrum and eigenfunctions for periodic boundary conditions also apply to the simple convection diffusion operator without randomness. The exceptional sensitivity to small perturbations of the spectrum for "absorbing" boundary conditions [27 can be understood in the following way: Since the operators we consider here are real, all eigenvalues must be real, or occur in complex conjugate pairs. If, in addition, we impose absorbing boundary conditions, we exclude all states carrying a real current. This exclusion implies real eigenfunctions which insures that all imaginary parts of the eigenvalues vanish for the lattice model in the limit $\eta=0$. Replacing this absorbing boundary condition by a weak link allows the eigenvalues to escape into the complex plane. The real spectrum studied in [27], considered as the $\eta \rightarrow 0$ limit of a lattice model, involves the simultaneous coincidence of all eigenvalues in pairs (except those at the band edge). This condition of extreme defectivity (an $N \times N$ matrix with only $\sim N / 2$ linearly independent eigenvectors !) is responsible for the exponential instabilities studied in [27.

Acknowledgements: It is a pleasure to acknowledge helpful conversations with P.W. Brouwer, A. Kudrolli, T. Neicu, Y. Oreg, and L. N. Trefethan. This work has been supported primarily by the Harvard Materials Research Science and Engineering Center through Grant No. DMR94-00396, and by the National Science Foundation through Grant No. DMR97-14725.

\section{APPENDIX A: ANALYTICAL RESULTS FOR THE SPECTRA IN THE HOMOGENEOUS SYSTEM}

We give here the expressions for the spectra of a 2 dimensional system with a homogeneous growth rate, computed for a square lattice, a triangular lattice, and the continuum model. For a homogeneous growth rate the eigenstates can be assumed to be plane waves, with $\mathbf{k}$-vectors in the first Brillouin zone. For simplicity we set the growth rate everywhere to zero. (A nonzero constant growth rate merely amounts to a constant shift of the eigenvalues $\Gamma(\mathbf{k})$.) For the square lattice one obtains

$$
\Gamma(\mathbf{k})=2 w \sum_{\nu=1}^{2}\left[\cosh \left(\mathbf{g} \cdot \mathbf{e}_{\nu}\right)\left(\cos \left(\mathbf{k} \cdot \mathbf{e}_{\nu}\right)-1\right)+i \sinh \left(\mathbf{g} \cdot \mathbf{e}_{\nu}\right) \sin \left(\mathbf{k} \cdot \mathbf{e}_{\nu}\right)\right] .
$$

For the triangular lattice the eigenvalues are

$$
\Gamma(\mathbf{k})=\frac{4 w}{3} \sum_{\nu=1}^{3}\left[\cosh \left(\mathbf{g} \cdot \mathbf{e}_{\nu}\right)\left(\cos \left(\mathbf{k} \cdot \mathbf{e}_{\nu}\right)-1\right)+i \sinh \left(\mathbf{g} \cdot \mathbf{e}_{\nu}\right) \sin \left(\mathbf{k} \cdot \mathbf{e}_{\nu}\right)\right],
$$

while for the continuum model the expression is simply 


$$
\Gamma(\mathbf{k})=-D k^{2}+i \mathbf{k} \cdot \mathbf{v}
$$

a form which agrees with the expansions of Eq. (A1) and Eq. (A2) for small wave vectors $\mathbf{k}$.

[1] Present Address: University of Illinois at Urbana Champaign, Department of Physics, 1110 W. Green Street, Urbana, IL 61801.

[2] J.-I. Wakita, K. Komatsu, A. Nakahara, T. Matsyama, and M. Matsushita, J. Phys. Soc. Japan 63, 1205 (1994); see also M. Matsushita, in Bacteria as Multicellular Organisms edited by J.A. Shapiro and M. Dworkin (Oxford University Press, Oxford, 1997).

[3] O. Rauprich, M. Matsushita, C.J. Weijer, F. Siegert, S.E. Esipov, and J.A. Shapiro, J. Bacteriology 178, 6525 (1996); J.A. Shapiro and D. Trubatch, Physica D 49, 214 (1991).

[4] E. Ben-Jacob, O. Schochet, A. Tenenbaum, I. Cohen, A. Czlrok and T. Vicsek, Nature 368, 46 (1994); E. Ben-Jacob, H. Shmueli, O. Shochet and A. Tenebaum, Physica A 187, 378 (1992) and Physica A 202, 1 (1994).

[5] E.O. Budrene and H. Berg, Nature 349, 630 (1991) and Nature 376, 49 (1995).

[6] J. D. Murray, Mathematical Biology, (Springer-Verlag, N.Y., 1993), Chapter 11.

[7] A.R. Robinson, Proc. R. Soc. Lond. A 453, 2295 (1997); see also R.V. Vincent and N.A. Hill, J. Fluid Mech. 327, 343 (1996).

[8] D.R. Nelson and N. Shnerb, Phys. Rev. E 58, 1383 (1998).

[9] For a moving mask, as in figure $\mathbb{1}$, the Fisher equation reads $\partial c\left(\mathbf{x}^{\prime}, t\right) / \partial t=D\left(\nabla^{\prime}\right)^{2} c\left(\mathbf{x}^{\prime}, t\right)+U\left(\mathbf{x}^{\prime}-\mathbf{v} t\right) c\left(\mathbf{x}^{\prime}, t\right)-b c^{2}\left(\mathbf{x}^{\prime}, t\right)$, where $\mathbf{x}^{\prime}$ is the coordinate in the laboratory frame where the growth medium is fixed. Upon transforming to new spatial coordinates $\mathbf{x}=\mathbf{x}^{\prime}-\mathbf{v} t$, in which the mask remains fixed, and then making the replacement $c(\mathbf{x}+\mathbf{v} t, t) \rightarrow c(\mathbf{x}, t)$, we obtain equation (1.1).

[10] K.A. Dahmen, D.R. Nelson, and N.M. Shnerb, to be published, Los Alamos Archive cond-mat/9807394.

[11] N. Shnerb and D.R. Nelson, Phys. Rev. Lett. 80, 5172 (1998).

[12] N. Hatano and D. R. Nelson, Phys. Rev. Lett. 77, 570 (1996); Phys. Rev. B 56, 8651 (1997); Phys. Rev. B 58, 8384 (1998).

[13] The term "mobility edge" is taken from the physics of disordered semiconductors, where it refers to an energy dividing localized from extended electron eigenfunctions. See B.I. Shklovskii and A.L. Efros Electronic Properties of Doped Semiconductors (Springer, Berlin, 1984).

[14] R.D. Richtmyer and K.W. Morton, Difference Methods for Initial Value Problems, Interscience Publishers (John Wiley) (1967).

[15] Piet Brouwer, private communication.

[16] V.G. Ganzha, E.V. Vorozhtsov, Numerical Solutions for Partial Differential Equations, Problem Solving Using Mathematica, CRC Press (1996).

[17] W.F. Ames, Numerical Methods for Partial Differential Equations, Academic Press (1992).

[18] W.H. Press, B.P. Flannery, S.A. Teukolsky, W.T. Vetterling Numerical Recipes (FORTRAN), Cambridge University Press, Cambridge (1990).

[19] P.W. Brouwer, P. G. Silvestrov and C. W. J. Beenakker, Phys. Rev. B, 56, R4333 (1997).

[20] E. Brezin and A. Zee, Nucl. Phys. B, 509[FS], 599 (1998); J. Feinberg and A. Zee, cond-mat/9710040.

[21] I. Y. Goldsheid and B. A. Khoruzhenko, Phys. Rev. Lett. 80, 2897 (1998).

[22] D. J. Thouless, J. Phys. C 5,77 (1972).

[23] See, e.g., F. R. Gantmacher, The Theory of Matrices, Chelsea Publishing Company, N.Y. (1974).

[24] The argument is best understood after taking the continuum limit by letting the lattice constant $\ell_{0}$ tend to zero. Suppose the continuum eigenfunction $\phi_{n}(x, g)$ with complex eigenvalue were in fact exactly zero at position $x=x_{0}$. By a similarity or "gauge" transformation similar to Eq. (4.4), one could shift all effects of the non-Hermiticity to the position $x_{0}$. The transformed eigenfunction would still vanish at $x_{0}$, because this gauge transformation is non-singular. All effects of nonHermiticity would then disappear for this eigenfunction, which would thus have to be localized with a real eigenvalue, leading to a contradiction. We are grateful to Ady Stern for discussions on this point.

[25] L.N. Trefethen, A. E.Trefethen, S. C. Reddy and T. A. Driscoll, Science 261578 (1993).

[26] L.N. Trefethen, SIAM Rev. 39383 (1997).

[27] S. C. Reddy and L. N. Trefethen, SIAM J. Appl. Math. 541634 (1994).

[28] The norm of a matrix $A$ is defined here as $\|A\| \equiv \sqrt{\text { max. eigenvalue of } A^{\dagger} A}$

[29] See, e.g., N. Hatano and D.R. Nelson, Phys. Rev. B 58, 8384 (1998).

[30] J. T. Chalker and B. Mehlig, cond-mat/9809090. 